\title{
LIOUVILLE EQUATION AND SPHERICAL CONVEX POLYTOPES
}

\author{
FENG LUO AND GANG TIAN
}

(Communicated by Peter Li)

\begin{abstract}
We study the Liouville equation $\Delta u=-e^{2 u}$ in the complex plane with prescribed singularities and obtain a necessary and sufficient condition for the existence of the solution. The proof is based on the continuity method and a uniqueness theorem.
\end{abstract}

Suppose $M$ is a punctured Riemann sphere with $n$ points $V_{1}, \ldots, V_{n}$ removed and $\alpha_{1}, \ldots, \alpha_{n} \in(0,2 \pi)$ are $n$ given numbers. We are interested in seeking a convex polytope with boundary $P$ in $S^{3}$ having $n$ vertices so that $P-\left\{V_{1}, \ldots, V_{n}\right\}$ is the Riemann surface $M$ and the cone angle at $V_{i}$ is $\alpha_{i}$. Our result is the following

Theorem 1. Let $M$ be an n-punctured Riemann sphere $(n \geq 3)$ and $\alpha_{i}$ be $n$ numbers in $(0,2 \pi)$. Then there is a (necessarily unique) convex polytope in $S^{3}$ with $n$ vertices whose boundary $P$ satisfies

(a) $P-\left\{V_{1}, \ldots, V_{n}\right\}$ is conformally equivalent to $M$ and

(b) the cone angle at $V_{i}$ is $\alpha_{i}$,

if and only if

$$
\sum_{i=1}^{n} \alpha_{i}>2 \pi(n-2)
$$

and

$$
\sum_{i \neq j} \alpha_{i}-\alpha_{j}<2 \pi(n-2), \quad \text { for all } j=1,2, \ldots, n .
$$

Since the cone angle of $|z|^{\alpha / 2 \pi-1}|d z|$ at 0 is $\alpha$, in terms of the singular metric, the theorem can be stated as

Theorem 2. The Liouville equation $\Delta u=-\exp (2 u)$ in the punctured complex plane $M=C-\left\{V_{1}, \ldots, V_{n}\right\}$ so that near each $V_{i}, u(z)=\beta_{i} \log \left|z-V_{i}\right|+$ a continuous function, where $\beta_{i} \in(-1,0)$ and $u=-2 \log |z|+a$ continuous

Received by the editors April 22, 1991.

1991 Mathematics Subject Classification. Primary 52A55; Secondary 53C25.

(c) 1992 American Mathematical Society $0002-9939 / 92 \$ 1.00+\$ .25$ per page 
function near the infinity, has a solution if and only if

(a) $\sum_{i=1}^{n} \beta_{i}>-2$ and

(b) $\left(\sum_{i \neq j} \beta_{i}\right)-\beta_{j}<0$, for all $j=1, \ldots, n$.

Furthermore, the solution is unique.

Remark 1. The existence of a solution of the Liouville equation under the conditions (a) and (b) was proven earlier by Troyanov.

Remark 2. The solution of the Liouville equation in general is not unique if the convexity condition $\beta_{i} \in(-1,0)$ is dropped. This was first observed by Troyanov. Indeed, take two copies of the standard 2-sphere and cut along geodesics of length $t \in(0, \pi)$. Then glue the two discs along the pairs of geodesics of length $t$. The result is a 2-sphere with two cone singularities of angles $4 \pi$. Clearly, for different $t$, the spherical metrics are not isometric. This construction also works for several punctures, which produces nonuniqueness of the solution of the Liouville equation with prescribed degenerations. On the other hand, the Liouville equations $\Delta u=e^{2 u}$ and $\Delta u=0$ corresponding to the hyperbolic and Euclidean metrics satisfy the maximal principal. Therefore, the uniqueness there is clear (see [Mc]). It is the uniqueness part that makes the proof nontrivial.

The organization of the paper is as follows. In $\S 1$ we prove the theorem assuming the uniqueness. In $\S 2$ we prove the uniqueness of the solution of the Liouville equation in the convex case.

\section{PROOF OF THE THEOREM}

By a theorem of Alexandrov, any spherical cone metric of positive curvature at the vertices is isometric to the boundary of a spherical convex polytope that may be degenerated to be the doubling of a spherical polyhedron. Furthermore, the corresponding spherical convex polytope is unique up to isometry of $S^{3}$. We are going to identify the spherical cone metric with the convex surface in $S^{3}$ in the following context.

Let us verify the necessity of the condition first.

Lemma 1. Given three numbers $\beta_{i} \in(0, \pi), i=1,2,3$, there is a unique (up to isometry) spherical triangle with inner angles $\beta_{i}$ 's if and only if the $\beta_{i}$ 's satisfy

$$
\sum_{i=1}^{3} \beta_{i}>\pi
$$

and

$$
\sum_{i \neq j} \beta_{i}-\beta_{j}<\pi, \quad \text { for } j=1,2,3 .
$$

Furthermore, equality in (4) holds if the triangle degenerates to a "lens", i.e., a triangle with three side lengths being $0, \pi$, and $\pi$.

Proof. By dualizing the triangle, we transform the problem into the following: given three numbers $\alpha_{i} \in(0, \pi), i=1,2,3$, there is a unique spherical 


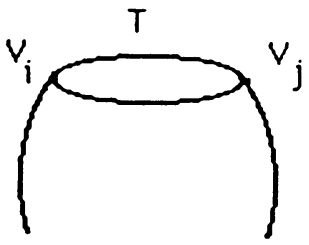

$P$ cut open along $T$
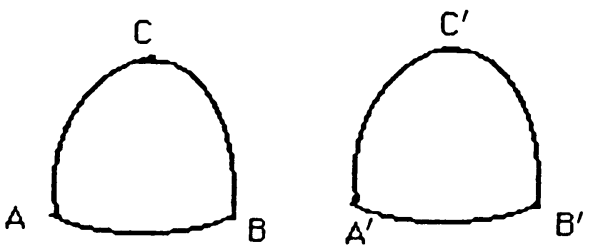

FIGURE 1

triangle with three side lengths $\alpha_{i}$ if and only if $\sum_{i=1}^{3} \alpha_{i}<2 \pi$ and $\sum_{i \neq j} \alpha_{i}>$ $\alpha_{j}$, for $j=1,2,3$. See Ryan [Ry] for a solution to this problem.

Now for any $n$-vertex convex polytope with boundary $P$ in $S^{3}$, construct a totally geodesic triangulation of $P$ so that 0 -simplexes are vertices of $P$. There are exactly $2(n-2)$ triangles and $3(n-2)$ edges in the triangulation by the Euler number calculation. Since the sum of the inner angles of each triangle is greater than $\pi$, we have $\sum_{i=1}^{n} \alpha_{i}>2 \pi(n-2)$. In fact the Gauss-Bonnet theorem says that the difference is the area of $P$. The second inequalities (2) are proven by induction on $n$. If $n=3$, then $P$ is a metric doubling of a spherical triangle, (2) follows from Lemma 1 . Assume it is true for $n$-vertex convex polytopes and $P$ is a polytope with $n+1$ vertices. We now apply the standard cutting and gluing technique of Alexandrov (see Busemann [Bu]) to reduce the number of vertices.

Let $T$ be the geodesic joining $V_{i}$ to $V_{j}$ in $P$. Construct two isometric spherical triangles $A B C$ and $A^{\prime} B^{\prime} C^{\prime}$ with base $|A B|=\left|A^{\prime} B^{\prime}\right|$ being the length of $T$ and inner angles $\angle C A B=\angle C^{\prime} A^{\prime} B^{\prime}=\pi-\alpha_{i} / 2$ and $\angle A B C=\angle A^{\prime} B^{\prime} C^{\prime}=$ $\pi-\alpha_{j} / 2$ (see Figure 1). We cut $P$ open along $T$ and glue the two triangles to the two sides of $T$ and the triangles to each other along $A C, A^{\prime} C^{\prime}, B C, B^{\prime} C^{\prime}$. This yields a spherical cone manifold of positive curvature with $n$ vertices since two vertices $V_{i}, V_{j}$ have cone angles $2 \pi$ by the construction. Let $\beta$ be the cone angle at the new vertex $C=C^{\prime}$. Applying Lemma 1 to the triangle $\mathrm{ABC}$, we have

$$
\left(\pi-\alpha_{i} / 2\right)+\pi>\beta / 2+\left(\pi-\alpha_{j} / 2\right)
$$

or $\alpha_{j}+2 \pi>\alpha_{i}+\beta$.

By the induction hypothesis, we have

$$
\beta+2 \pi(n-2)>\sum_{k \neq i, j} \alpha_{k}
$$

The sum of these inequalities gives the inequalities (2) for $P$.

To verify that the condition is also sufficient, let $\mathscr{P}_{n}$ be the space of all boundaries of labeled $n$-vertex convex polytopes in $S^{3}$ modulo isometry, or the same, the space of all spherical cone metrics of positive curvature with $n$ cones modulo isometry. The topology on $\mathscr{P}_{n}$ is induced by the Hausdorff convergence. Let $\mathscr{M}_{n}$ be the space of all labeled $n$-punctured Riemann spheres and let $\mathscr{A}$ be the convex set $\left\{\left(\alpha_{1}, \alpha_{2}, \ldots, \alpha_{n}\right) \mid \alpha_{i}\right.$ 's $\in(0,2 \pi)$ and satisfy (1) 
and (2)\}. There is a natural map

$$
\Pi: \mathscr{P}_{n} \rightarrow \mathscr{M}_{n} \times \mathscr{A}
$$

obtained by recording the conformal structure of the metric and the cone angles. We will show that $\Pi$ is a homeomorphism and hence the theorem. Clearly, $\Pi$ is continuous with respect to the natural topologies in these spaces.

Step 1. $\mathscr{P}_{n}$ and $\mathscr{M}_{n} \times \mathscr{A}$ are manifolds, and $\operatorname{dim} \mathscr{P}_{n}=3(n-2)=\operatorname{dim} \mathscr{M}_{n}+$ $\operatorname{dim} \mathscr{A}$. Let $P \in \mathscr{P}_{n}$. We construct a totally geodesic triangulation $K$ of $P$ with 0 -simplexes the vertices of $P$. Then, there are $3(n-2)$ edges in $K$. A slight change of the lengths of these edges still produces a spherical cone metric of positive curvature. Furthermore all the resulting cone metrics are distinct (up to isometry). Hence $\mathscr{P}_{n}$ is a manifold of dimension $3(n-2)$. Clearly, $\operatorname{dim} \mathscr{M}_{n}=2 n-6$ and $\operatorname{dim} \mathscr{A}=n$.

Step 2. $\Pi$ is injective. This is the theorem to be proved in $\S 2$.

Step 3. $\Pi$ is a closed map. Because $\mathscr{M}_{n} \times \mathscr{A}$ is connected, the above assertion implies $\Pi$ is a homeomorphism.

Let $Q^{m}$ be a sequence of elements in $\mathscr{P}_{n}$ so that $\Pi\left(Q^{m}\right)$ converges to a point in $\mathscr{M}_{n} \times \mathscr{A}$ with second coordinate $\alpha$ in $\mathscr{A}, \alpha=\left(\alpha_{1}, \alpha_{2}, \ldots, \alpha_{n}\right)$. We will show that $Q^{m}$ has a convergent subsequence in $\mathscr{P}_{n}$.

By compactness of compact sets in $S^{3}$ in the Hausdorff metric, we may assume $Q^{m}$ converges in the Hausdorff metric to a convex polytope $Q$ in $S^{3}$. Furthermore, since there are only finitely many triangulations with $n$ vertices, the totally geodesic triangulations of $Q^{m}$ constructed above can be assumed to be isomorphic (as 2-complex) to a triangulation $K$ of $S^{2}$. The goal is to show that $Q$ has exactly $n$ vertices.

Let $V_{i}$ and $\Delta_{j}$ be the 0 -simplexes and 2-simplexes in $K$; let $V_{i}^{m}$ and $\Delta_{j}^{m}$ be the corresponding vertex and triangle in $Q^{m}$; and let $\alpha_{i}^{m}$ be the cone angle of $V_{i}^{m}$ in $Q^{m}$. The spherical distance between $a$ and $b$ in the cone manifold is denoted by $d(a, b)$.

By hypothesis, $\lim \alpha_{i}^{m}=\alpha_{i}$ for $i=1,2, \ldots, n$ and $\alpha=\left(\alpha_{1}, \alpha_{2}, \ldots, \alpha_{n}\right) \in$ $\mathscr{A}$, the area of $Q$ is the limit of the area of $Q^{m}$ that is bounded below. Indeed, $\operatorname{area}(Q)=\sum_{i=1}^{n} \alpha_{i}-2 \pi(n-2)>0$. Hence, $Q$ is not a point or a line segment. This shows $\lim d\left(V_{i}^{m}, V_{j}^{m}\right)>0$ for some $i \neq j$.

Case (I). $\lim \left(V_{i}^{m}, V_{j}^{m}\right)>0$ for all $i \neq j$, then $Q$ has $n$ vertices because no collapsing between vertices occurs in the limit and each $\alpha_{i}$, being the cone angle of $\lim V_{i}^{m}$ in $Q$, is in $(0,2 \pi)$. Hence $Q \in \mathscr{P}_{n}$.

Case (II). There are two vertices that collapse in the limit, say,

$$
\lim d\left(V_{1}^{m}, V_{2}^{m}\right)=0 \text {. }
$$

Then, we have

Claim. There is exactly one $i$, so that

$$
\lim d\left(V_{1}^{m}, V_{i}^{m}\right)>0 \text { and } \lim d\left(V_{1}^{m}, V_{j}^{m}\right)=0 \text { for all } j \neq i \text {. }
$$

Otherwise, say $\lim d\left(V_{1}^{m}, V_{j}^{m}\right)>0$ for $j \neq i$. Consider the set of all embeddings of open annuli $A$ in $Q^{m}-\left\{V_{1}^{m}, \ldots, V_{n}^{m}\right\}$ so that $V_{1}^{m}, V_{2}^{m}$ lie in 
one complement of $A$ in $Q^{m}$ and $V_{i}^{m}$ and $V_{j}^{m}$ lie in the other complement. Now the maximum modulus of these $A$ will be arbitrarily large as $m$ tends to infinity since $V_{1}^{m}$ and $V_{2}^{m}$ tends to the same point in the limit. On the other hand, the conformal structures of $Q^{m}$ lie in a compact set in $\mathscr{M}_{n}$ due to the convergent. This implies there is an upper bound on the modulus of such annuli. The contradiction establishes the assertion of the claim.

Let us assume that $i=3$ in the claim for simplicity. $Q$ has only two vertices in this case. Therefore $Q$ is the suspension of a convex polyhedron in $S^{2}$ between two antipodal points.

Let $\Delta_{i}$ 's, $i=1,2, \ldots, l$, be the triangles incident to $V_{3}$ in $K$ and $\Delta_{j}$ 's, $j=l+1, \ldots, 2(n-2)$, be the rest of the triangles. The above discussion shows that $\Delta_{i}^{m}$ 's tend to a lens, i.e., degenerate triangles with side lengths $\pi$, $\pi, 0$ and the $\Delta_{j}^{m}$ 's tend to a point. Let the inner angles of $\Delta_{k}^{m}$ be $\alpha_{1 k}^{m}, \alpha_{2 k}^{m}$, and $\alpha_{3 k}^{m}$. For each $\Delta_{i}^{m}, i=1,2, \ldots, l$, say $\alpha_{3 i}^{m}$ is the inner angle incident to $V_{3}^{m}$. By Lemma $1, \alpha_{3 i}^{m}+\pi>\alpha_{2 i}^{m}+\alpha_{1 i}^{m}$, which becomes equality in the limit as $m$ tends to infinity. For each $\Delta_{j}^{m}, j \geq l+1$, we have $\pi<\alpha_{1 j}^{m}+\alpha_{2 j}^{m}+\alpha_{3 j}^{m}$. It becomes equality as $m$ tends to infinity, since the difference is the area of the triangle. Taking the limit and considering the sum of all these equalities, we have

$$
\alpha_{3}+2 \pi(n-2)=\sum_{i \neq 3} \alpha_{i}
$$

This contradicts the assumption that $\alpha \in \mathscr{A}$.

Remark 3. With a slight modification of the proof, one can also show the corresponding theorem for zero curvature and -1 curvature. The uniqueness of the solution follows easily from the maximal principle. The conditions are $\sum_{i=1}^{n} \alpha_{i}=2 \pi(n-2)$ and $\sum_{i=1}^{n} \alpha_{i}<2 \pi(n-2)$, respectively. The results in these cases are well known, see Hulin and Troyanov [HT], McOwen [Mc], and Thurston [Th]. It is interesting to note that the second set of inequalities (2) are automatically satisfied in these cases.

\section{Proof of the UniQueness theorem}

Let $p_{1}, \ldots, p_{n}(n \geq 3)$ be distinct points in $S^{2}$. For any fixed $\left(\alpha_{1}, \ldots, \alpha_{n}\right)$ $\in R^{n}$, an $\left(\alpha_{1}, \ldots, \alpha_{n}\right)$-weighted metric $g$ on $S^{2}$ is a conformal metric on $S^{2} \backslash\left\{p_{1}, \ldots, p_{n}\right\}$ such that for each $i$ between 1 and $n$, let $z$ be the local conformal coordinate of $S^{2}$ at $p_{i}$,

$$
g=a|z|^{2 \alpha_{i}}|d z|^{2}
$$

where $a$ is a smooth positive function near $p_{i}$.

From now on, all $\alpha_{i}$ are in $(-1,0]$. An $\left(\alpha_{1}, \ldots, \alpha_{n}\right)$-weighted metric $g$ on $S^{2}$ is said spherical if the scalar curvature of $g$ is constant outside $p_{1}, \ldots, p_{n}$. We always normalize a spherical metric on $S^{2}$ such that the scalar curvature is one. Obviously, there is a necessary condition for the existence of a spherical metric on $S^{2}$ with weights $\left(\alpha_{1}, \ldots, \alpha_{n}\right)$, namely,

$$
-\sum_{i=1}^{n} \alpha_{i}<1
$$


This follows from the Gauss-Bonnet Theorem.

Each $\left(\alpha_{1}, \ldots, \alpha_{n}\right)$-weighted metric $g$ has an associated cohomology class $\omega_{g}$ in $H^{2}\left(S^{2}, R\right)$, i.e., the Kähler form or the volume form of $g$.

Lemma 2. Let $g_{1}, g_{2}$ be two $\left(\alpha_{1}, \ldots, \alpha_{n}\right)$-weighted metrics such that their associated Kähler forms are cohomological to each other. Then there is a bounded function $\psi$, unique to addition of a constant, such that

$$
\omega_{g_{2}}=\omega_{g_{1}}+\frac{\sqrt{-1}}{2 \pi} \partial \bar{\partial} \psi
$$

where $\partial, \bar{\partial}$ are the $\partial$-operator and $\bar{\partial}$-operator of the conformal structure of $S^{2}$. Proof. This is the well-known $\partial \bar{\partial}$ lemma for Kähler manifolds. Here $\omega_{1}$ and $\omega_{2}$ have singularities at $p_{1}, \ldots, p_{n}$. But they do not yield extra difficulties.

The purpose of this section is to prove the uniqueness of the spherical metrics with fixed weights.

Theorem 3. Let $n \geq 3$ and $\left(\alpha_{1}, \ldots, \alpha_{n}\right) \in(-1,0)^{n}$. Then there is at most one normalized spherical metric on $S^{2}$ with given weights at $p_{1}, \ldots, p_{n}$.

Let $g_{1}, g_{2}$ be the two normalized spherical metrics with weights at $p_{1}, \ldots$, $p_{n}$. Then if $R\left(g_{1}\right)$ and $R\left(g_{2}\right)$ are the curvature forms of $g_{1}, g_{2}$,

$$
R\left(g_{i}\right)=\omega_{i}, \quad i=1,2,
$$

where $\omega_{i}$ are Kähler forms of $g_{i}$. By the Gauss-Bonnet Theorem, $R\left(g_{1}\right)$ is cohomological to $R\left(g_{2}\right)$ so there is a continuous function $\psi$ such that

$$
\omega_{2}=\omega_{1}+\frac{\sqrt{-1}}{2 \pi} \partial \bar{\partial} \psi
$$

Let $\Delta_{i}$ be the laplacian operators of $g_{i}(i=1,2)$. Then

$$
\omega_{2} / \omega_{1}=1+\Delta_{1} \psi
$$

It follows from (6) that

$$
1+\Delta_{1} \psi=e^{-\psi} \text { on } S^{2} .
$$

We should point out that this equation is in the sense of distribution, since $\psi$ is not $C^{2}$-smooth in general at $p_{1}, \ldots, p_{n}$.

Let $g$ be an $\left(\alpha_{1}, \ldots, \alpha_{n}\right)$-weighted metric on $S^{2}$. Consider the following eigenvalue problem:

$$
\lambda_{1}(g)=\inf \left\{\int_{S^{2}}|\nabla \psi|_{g}^{2} \omega_{g} \mid \int_{S^{2}} \psi^{2} \omega_{g}=1, \int_{S^{2}} \psi \omega_{g}=0\right\} .
$$

The infimum of (10) is attained by the continuous solution of the equation

$$
-\Delta_{g} \psi=\lambda_{1}(g) \psi
$$

where $\Delta_{g}$ is the laplacian of $g$. This can be easily proved by standard methods. The infimum $\lambda_{1}(g)$ is said to be the first nonzero eigenvalue of $\Delta_{g}$. 
Lemma 3. If $n \geq 3$ and $g$ is a conformal metric such that $\omega_{g}=\omega_{1}+\frac{\sqrt{-1}}{2 \pi} \partial \bar{\partial} \psi_{t}$ where

$$
1+\Delta_{1} \psi_{t}=e^{t \psi_{t}} \quad \text { on } S^{2}
$$

with $0 \leq t \leq 1$, then $\lambda_{1}(g)>t$.

Proof. The curvature form

$$
\begin{aligned}
R(g) & =-\frac{\sqrt{-1}}{2 \pi} \partial \bar{\partial} \log \frac{\omega_{g}}{\omega_{1}}+R\left(g_{1}\right)=t \frac{\sqrt{-1}}{2 \pi} \partial \bar{\partial} \psi_{t}+\omega_{1} \\
& =(1-t) \omega_{1}+t \omega_{g} \geq t \omega_{g} \quad \text { on } S^{2} \backslash\left\{p_{1}, \ldots, p_{n}\right\}
\end{aligned}
$$

and the equality holds only if $t=1$. A direct computation shows: if $\psi$ is the eigenfunction of $\Delta_{g}$, with eigenvalue $\lambda_{1}(g)$, then

$$
\begin{aligned}
\Delta_{g}\left(|\nabla \psi|_{g}^{2}\right)= & \frac{R(g)}{\omega_{g}}|\nabla \psi|_{g}^{2}-2 \lambda_{1}(g)|\nabla \psi|_{g}^{2}+\lambda_{1}(g)^{2} \psi^{2} \\
& +\left|\nabla^{(1,0)} \partial \psi\right|_{g}^{2} \quad \text { in } S^{2} \backslash\left\{p_{1}, \ldots, p_{n}\right\}
\end{aligned}
$$

where $|\cdot|_{g}$ means the norm with respect to $g$ and $\nabla^{(1,0)} \partial \psi$ is the $(2,0)$-part of the Hessian of $\psi$. Therefore, if we can prove $\int_{S^{2}} \Delta_{g}\left(|\nabla \psi|_{g}^{2}\right) \omega_{g}=0$, then

$$
\int_{S^{2}}\left(\lambda_{1}^{2}(g) \omega_{g}-\lambda_{1}(g) R(g)\right)=\int_{S^{2}}\left|\nabla^{(1,0)} \partial \psi\right|_{g}^{2} \omega_{g}
$$

It implies $\lambda_{1}(g)>t$ if $t<1$ and $\lambda_{1}(g) \geq 1$ if $t=1$; moreover, if $\lambda_{1}(g)=1$ when $t=1$, then

$$
\int_{S^{2}}\left|\nabla^{(1,0)} \partial \psi\right|_{g}^{2} \omega_{g}=0
$$

i.e., $X^{i}=g^{i \bar{j}} \psi_{\bar{j}}$ defines a holomorphic vector field on $S^{2} \backslash\left\{p_{1}, \ldots, p_{n}\right\}$. On the other hand, since

$$
\int_{S^{2} \backslash\left\{p_{1}, \ldots, p_{n}\right\}}|X|_{g}^{2} \omega_{g}=\int_{S^{2}}|\nabla \psi|_{g}^{2} \omega_{g}=\lambda_{1}(g)=1
$$

and the metric $g=g_{2}$ is bounded from below by some euclidean metric near each $p_{i}$. $X$ can be extended to be a holomorphic vector field on $S^{2}$.

Fix a $p_{i}(i=1,2, \ldots, n)$, near $p_{i}, g=a|z|^{2 \alpha_{i}}|d z|^{2}=a|z|^{2 \alpha_{i}} g_{0}$ in local coordinate $z$, then

$$
-\Delta_{0} \psi=a|z|^{2 \alpha_{i}} \psi
$$

where $\Delta_{0}$ is the standard laplacian operator in a neighborhood of $p_{i}$. Since $\psi$ is bounded, $\Delta_{0} \psi$ is $L^{q}$-bounded for any $q<-1 / \alpha_{i}$, consequently, if $-2 \alpha_{i} \geq 1$ then $|\nabla \psi|_{g_{0}}$ is $L^{q}$-bounded with respect to $g_{0}$ for any $q<2 /\left(-2 \alpha_{i}-1\right)$, and if $-2 \alpha_{i}<1$ then $\nabla \psi$ is continuous. Since $|X|_{g}^{2}=|\nabla \psi|_{g}^{2}, X\left(p_{i}\right)=0$ if $-2 \alpha_{i}<1$. If $-2 \alpha_{i} \geq 1$, since $|X|_{g}^{2} \omega_{g}$ is the same as $a^{2}|z|^{-4 \alpha_{i}}|X|_{g_{0}}^{2} \omega_{g_{0}}$, it follows from the $L^{2}$-finiteness of $|X|_{g}$ that $X\left(p_{i}\right)=0$. Thus we have proved that $X$ vanishes at $p_{i}, \ldots, p_{n}$. Since $n \geq 3, X \equiv 0$. This contradicts that $\psi$ is nonconstant, so $\lambda_{1}(g)>1$. 
It remains to show $\int_{S^{2}} \Delta_{g}\left(|\nabla \psi|_{g}^{2}\right) \omega_{g}=0$. Choose a local conformal coordinate $z$ such that $g=a|z|^{2 \alpha_{i}}|d z|^{2}=a|z|^{2 \alpha_{i}} g_{0}$. Then

$$
-\Delta_{0} \psi=\lambda_{1} a|z|^{2 \alpha_{i}} \psi \text {. }
$$

Put $r=|z|, \rho=-\log r$, i.e., $r=e^{-\rho}$. Then (15) becomes

$$
\frac{\partial^{2} \psi}{\partial \rho^{2}}+\frac{\partial^{2} \psi}{\partial \theta^{2}}=\lambda_{1} a e^{-2\left(1+\alpha_{i}\right) \rho} \psi
$$

and

$$
\int_{\substack{c \leq \rho<\infty \\ 0 \leq \theta \leq 2 \pi}}\left(\left|\frac{\partial \psi}{\partial \rho}\right|^{2}+\left|\frac{\partial \psi}{\partial \theta}\right|^{2}\right) d \rho d \theta<+\infty
$$

where $c$ is some nonnegative number, for simplicity, set $c=0$. It follows from (16) that

$$
\frac{d}{d \rho} \int_{0}^{2 \pi} \frac{\partial \psi}{\partial \rho}\left(e^{-\rho}, \theta\right) d \theta=\lambda_{1} e^{-2\left(1+\alpha_{i}\right) \rho} \int_{0}^{2 \pi} a \psi\left(e^{-\rho}, \theta\right) d \theta ;
$$

therefore,

$$
\int_{0}^{2 \pi} \frac{\partial \psi}{\partial \psi}\left(e^{-\rho}, \theta\right) d \theta=\lambda_{1} \int_{\infty}^{\rho} e^{-2\left(1+\alpha_{i}\right) t} d t \int_{0}^{2 \pi} a \psi\left(e^{-t}, \theta\right) d \theta+C_{1} .
$$

By (17), $C_{1}=0$ and

$$
\lim _{\rho \rightarrow+\infty}\left(e^{2\left(1+\alpha_{i}\right) \rho} \int_{0}^{2 \pi} \frac{\partial \psi}{\partial \rho}\left(e^{-\rho}, 0\right) d \theta\right)=\lambda_{1} a \psi(0,0) .
$$

Taking the derivative on both sides of (16) on $\rho$, we have

$$
\begin{aligned}
& \left(\frac{\partial^{2}}{\partial \rho^{2}}+\frac{\partial^{2}}{\partial \theta^{2}}\right)\left(\frac{\partial \psi}{\partial \rho}\right) \\
& \quad=\lambda_{1} a e^{-2\left(1+\alpha_{i}\right) \rho} \frac{\partial \psi}{\partial \rho}-2\left(1+\alpha_{i}\right) \lambda_{1} a e^{-2\left(1+\alpha_{i}\right) \rho} \psi+O\left(e^{-\left(3+2 \alpha_{i}\right) \rho}\right)
\end{aligned}
$$

where $O\left(e^{-\mu \rho}\right)$ denotes a quantity of order $e^{-\mu \rho}$.

Multiplying both sides of (20) by $\partial \psi / \partial \rho$, integrating by parts, and making use of the Schwartz inequality, we obtain

$$
\int_{\substack{\rho_{0} \leq \rho<\infty \\ 0 \leq 0 \leq 2 \pi}}\left|\nabla\left(\frac{\partial \psi}{\partial \rho}\right)\right|^{2} d \rho d \theta=e^{-2\left(1+\alpha_{i}\right) \rho_{0}}\left(\left(\int_{\rho \geq \rho_{0}}|\nabla \psi|^{2} d \rho d \theta\right)^{1 / 2}+O\left(e^{-\rho_{0}}\right)\right) .
$$

By the Poincare inequality,

$$
\int_{\substack{\rho_{0} \leq \rho<\infty \\ 0 \leq 0 \leq 2 \pi}}\left|\frac{\partial \psi}{\partial \rho}-\frac{1}{2 \pi} \int_{0}^{2 \pi} \frac{\partial \psi}{\partial \rho} d \tilde{\theta}\right|^{2} d \rho d \theta=e^{-2\left(1+\alpha_{i}\right) \rho_{0}} O(1)
$$


where $o(1)$ denotes a quantity converging to zero as $\rho \rightarrow+\infty$. Then using the equation (20) and the standard elliptic estimates, one can show

$$
e^{\left(1+\alpha_{i}\right) \rho}\left(\frac{\partial \psi}{\partial \rho}-\frac{1}{2 \pi} \int_{0}^{2 \pi} \frac{\partial \psi}{\partial \rho}\left(e^{-\rho}, \theta\right) d \theta\right) \rightarrow 0 \text { as } \rho \rightarrow+\infty .
$$

Consequently,

$$
\lim _{\rho \rightarrow+\infty} e^{\left(1+\alpha_{i}\right) \rho}\left|\frac{\partial \psi}{\partial \rho}\right|=0
$$

Similarly,

$$
\lim _{\rho \rightarrow+\infty} e^{\left(1+\alpha_{i}\right) \rho}\left|\frac{\partial \psi}{\partial \theta}\right|=0 .
$$

Equations (21) and (22) together imply

$$
\lim _{r \rightarrow 0}|\nabla \psi|_{g}^{2}=0
$$

Then it follows easily from (23) that

$$
\int_{S^{2}} \Delta_{g}\left(|\nabla \psi|_{g}^{2}\right) \omega_{g}=0 .
$$

Corollary 1. Assume $n \geq 3$. Define I to be the subinterval in $[0,1]$ such that if $t \in I$, then $(12)_{s}$ has a bounded solution $\psi_{s}$ for any $1 \geq s \geq t$ satisfying $\int_{S^{2}}\left|\nabla \psi_{S}\right|_{g_{s+1}}^{2} \omega_{s+1}<\infty$ where $g_{s+1}$ is the metric on $S^{2}$ with Kähler form $\omega_{1}+$ $(\sqrt{-1} / 2 \pi) \partial \bar{\partial} \psi_{s}$. Then I is an open interval in $[0,1]$.

Proof. This follows from Lemma 3 and the Implicit Function Theorem in suitable weighted Sobolev spaces.

By the choice of $\psi_{1}=\psi$, we know $I \neq \varnothing$, i.e., $1 \in I$. If we can prove $I$ is closed, then $0 \in I$. By the maximum principle for Laplacian operator on $S^{2}$, the solution of $(12)_{0}$ is unique, i.e., the trivial solution zero. Then by Lemma 3 and Implicit Function Theorem, the solutions of $(12)_{t}$ for $t$ small are unique and so are zeros. Continuing such an argument until $t=1$, we can prove that $\psi_{1}=\psi=0$. Therefore, $g_{2}=g_{1}$, i.e., the theorem is proved.

Now it remains to prove that $I$ is closed. Let $\left\{\psi_{t}\right\}_{t_{0}<t \leq 1}$ be a family of solutions of $(12)_{t}$; then by Lemma 3 , it is a smooth family.

Lemma 4. Let $\left\{\psi_{t}\right\}_{t_{0}<t \leq 1}$ be the family of the solutions of $(12)_{t}$ as above. Then

$$
\frac{d}{d t}\left(\int_{S^{2}}\left|\nabla \psi_{t}\right|_{g_{t+1}}^{2} \omega_{t+1}\right) \geq 0
$$

where $\psi_{t}=\partial \psi_{t} / \partial t$.

Proof.

$$
\begin{aligned}
\frac{d}{d t}\left(\int_{S^{2}}\left|\nabla \psi_{t}\right|_{g_{t+1}^{2}} \omega_{t+1}\right) & =\frac{d}{d t}\left(\int_{S^{2}}\left|\nabla \psi_{t}\right|_{g_{1}}^{2} \omega_{1}\right) \\
& =-2 \int_{S^{2}}\left(\Delta_{1} \dot{\psi}_{t}\right) \psi_{t} \omega_{1}=-2 \int_{S^{2}}\left(\Delta_{t+1} \dot{\psi}_{t}\right) \psi_{t} \omega_{t+1}
\end{aligned}
$$


Differentiating $(12)_{t}$ on $t$, we have

$$
\Delta_{1} \dot{\psi}_{t}=-e^{-t \psi_{t}}\left(t \dot{\psi}_{t}+\psi_{t}\right),
$$

i.e., $\Delta_{t+1} \dot{\psi}_{t}+t \dot{\psi}_{t}=-\psi_{t}$. Applying this to (25) yields

$$
\frac{d}{d t}\left(\int_{S^{2}}\left|\nabla \psi_{t}\right|_{g_{t+1}}^{2} \omega_{t+1}\right)=2 \int_{S^{2}}\left(\Delta_{t+1} \dot{\psi}_{t}\right)\left(\Delta_{t+1} \dot{\psi}_{t}+t \dot{\psi}_{t}\right) \omega_{t} .
$$

Then (24) follows from Lemma 3.

Corollary 2. Let $\left\{\psi_{t}\right\}_{t_{0}<t \leq 1}$ be as in Lemma 4. Then for $t>t_{0}$,

$$
\int_{S^{2}}\left|\nabla \psi_{t}\right|_{g^{1}}^{2} \omega_{1} \leq \int_{S^{2}}\left|\nabla \psi_{1}\right|_{g_{1}}^{2} \omega_{1}<\infty
$$

namely, for $t>t_{0}$,

$$
\int_{S^{2}} \psi_{t}\left(\omega_{1}-\omega_{t}\right) \leq \int_{S^{2}}\left|\nabla \psi_{1}\right|_{g_{1}}^{2} \omega_{1}<\infty
$$

By $(12)_{t}, \Delta_{1} \psi_{t}+1 \geq 0$, i.e., $\psi_{t}$ is almost subharmonic, so the Moser iteration yields

$$
\sup _{S^{2}} \psi_{t} \leq C\left(1+\int_{S^{2}} \psi_{t} \omega_{1}\right)
$$

where $C$ is a uniform constant. On the other hand, since $R\left(g_{t+1}\right)>t$, the Sobolev constant of $g_{t+1}$ is uniformly bounded. Also, the equation (12) implies $\Delta_{t+1} \psi_{t}+1 \leq 0$. Therefore a Moser iteration yields

$$
-\inf _{S^{2}} \psi_{t} \leq C\left(1-\int_{S^{2}} \psi_{t} \omega_{t+1}\right)
$$

so $\left|\psi_{t}\right|_{C^{0}}$ are uniformly bounded for $t_{0}<t \leq 1$. Then the standard elliptic theory implies that a subsequence of $\left\{\psi_{t}\right\}_{t_{0}<t \leq 1}$ converges to a bounded solution $\psi_{t_{0}}$ of $(12)_{t_{0}}$ with $\int_{S^{2}}\left|\nabla \psi_{t_{0}}\right|_{g_{t_{0}+1}}^{2} \omega_{t_{0}+1}<\infty$. Such a convergence is actually in at least $C^{3}$-norm outside the singular points $p_{1}, \ldots, p_{n}$.

It follows that $I$ is closed.

\section{ACKNOWLEDGMENT}

The first author would like to thank Richard Stong for stimulating discussions that led him to recognize the inequalities. He also thanks $\mathrm{S}$. A. Chang, M. Troyanov, and the referee for useful comments.

\section{REFERENCES}

[Bu] H. Busemann, Convex surfaces, Interscience, New York, 1958.

[HT] D. Hulin and M. Troyanov, Prescribing curvature on open surfaces, preprint. 
[Mc] R. McOwen, Point singularities and conformal metrics on Riemann surfaces, Proc. Amer. Math. Soc. 103 (1988), 222-224.

[Ry] P. Ryan, Euclidean and non-Euclidean geometry, Cambridge Univ. Press, New York, 1986.

[Th] W. Thurston, Shape of polyhedrons, preprint.

Department of Mathematics, University of California, Los ANGeles, California 90024 E-mail address: luo@math.ucla.edu

Courant Institute of Mathematics Science, New York, New York 10012

E-mail address: tian@math1.nyu.edu 